\title{
Strengthening the referral Chain and providing one window diabetes eye care facility to people with Type-2 Diabetes: A six-year follow-up study from Pakistan
}

\author{
Shahid Ahsan', Muhammad Saleh Memon², Muhammad Faisal Fahim³, \\ Tauseef Mahmood ${ }^{4}$, Sikander Ali Sheikh
}

\begin{abstract}
Objective: To report the results of implementation of two-pronged system for strengthening of referral and receiving end of referral chain for people with Type-2 diabetes mellitus (T2DM) at a tertiary eye care hospital in Karachi.

Methods: This observational, cross sectional study was conducted from the data collected in "Strengthening Pakistan's response to Diabetic Retinopathy" project. Peripheral referral was improved through community awareness camps $(n=48)$, refreshing knowledge of primary healthcare professionals (PHCP) and conducting retinopathy screening camps $(n=85)$ in the community. T2DM patients with best corrected visual acuity $(B C V A)<6 / 18$ or had retinopathy sign on screening were referred to tertiary center. The receiving end of the referral was improved by establishing "one window facility" at tertiary eye care center. Facility consisted of eight stations starting from registration, visual assessment, fundus photographs, consultation with ophthalmologist, anthropometry measurement, consultation with diabetologist to finally meeting with diabetes educator. At every station, patient's information was directly entered in HIMS software.

Results: A total of 50,595 patients attended tertiary center over six years. Among all $34685(68.5 \%)$ were new registration and 15910 (31.4\%) were follow ups. During first year (2014-15) out of total registered individuals with DM, newly registered were $4414(89.5 \%)$ and $518(10.5 \%)$ were follow-ups. In the final year (2019-20) new cases registered reached to 62\% ( $n=7916)$ with $38 \%(n=4852)$ follow-ups. Patients referred by PHCP increased from 6.5\% in 2014-15 to 43.7\% in 2019-20. An increased uptake of all treatment modalities for retinopathy like laser (increased by 16.76\%), intra-vitreal injections (by 14.72\%) and vitrectomy (by $51.47 \%)$ were also observed.

Conclusion: Implementation of two prong system resulted in improved service uptake, better referral system, enhanced follow-ups and increased intervention uptake.
\end{abstract}

KEYWORDS: Comprehensive eye care, Diabetic retinopathy, Referral source, Type-2 Diabetes Mellitus.

doi: https://doi.org/10.12669/pjms.37.7.3946

How to cite this:

Ahsan S, Memon MS, Fahim MF, Mahmood T, Sheikh SA. Strengthening the referral Chain and providing one window diabetes eye care facility to people with Type-2 Diabetes: A six-year follow-up study from Pakistan. Pak J Med Sci. 2021;37(7):1837-1842. doi: https://doi.org/10.12669/pjms.37.7.3946

This is an Open Access article distributed under the terms of the Creative Commons Attribution License (http://creativecommons.org/licenses/by/3.0), which permits unrestricted use, distribution, and reproduction in any medium, provided the original work is properly cited.

\section{Correspondence:}

Dr. M. Saleh Memon FRCS(Eden),

Director Research,

Al Ibrahim Eye Hospital,

ISRA Postgraduate Institute of Ophthalmology,

Karachi, Pakistan.

Email: salehmemon@yahoo.com

* Received for Publication:

* Revision Received:

* Revision Accepted:
November 26, 2020

May 10,2021

June 15, 2021

\section{INTRODUCTION}

Chronic complications of Type-2 DM have insidious onset. ${ }^{1}$ Once developed; it remains unnoticed till the disease is well advanced to produce symptoms. International organizations therefore recommend annual screening of these complications for tertiary prevention of diabetic complications. Early detection and prompt treatment halts or even regresses the progression 
of this complications. ${ }^{2}$ Despite recommendations and documented benefits, uptake of screening facilities in developing countries has yet not reached the optimal levels. ${ }^{3}$ Low uptake of health care services is a general phenomenon in developing countries. ${ }^{4}$ According to WHO only a quarter of people in need of eye care, use eye care services. ${ }^{5}$

Reasons for low uptake of health facilities vary from country to country and region to region. In low and middle-income countries like Pakistan, people in general have poor education and low health literacy. There is generally a lack of awareness about importance of screening. The incongruous health beliefs and misconceptions of the community also affect their attitude towards uptake of services. This situation further lowers the acceptance of appropriate treatment after screening and causes loss to follow up. These barriers along with low or inappropriate referral, un-even distribution of skilled personnel, overburdened specialists in tertiary centers and inadequate resources in higher health care facilities are the challenges for the provision of appropriate post screening treatment. ${ }^{3}$

With escalating prevalence of diabetes worldwide, $^{6}$ screening of chronic diabetic complications, particularly retinopathy screening has become a matter of concern for public health workers since beginning of the twentieth century. ${ }^{3}$ In the developed countries, diabetic retinopathy (DR) has emerged as one of the major causes of vision impairment. ${ }^{7}$ In Pakistan, uncorrected refractive errors, cataract, and glaucoma, ${ }^{8}$ once surpassed retinopathy (DR) as common conditions of vision impairment and blindness. Now it is widely realized that in the last decade the frequency of patients being diagnosed with diabetes and subsequently with DR has increased incrementally in the country. ${ }^{9}$ Recent survey has shown that prevalence of diabetes in Pakistan is $26.3 \%$, of which $19.2 \%$ are those with known DM. ${ }^{9}$ Among patients with DM, studies have reported that $20-26 \%$ have retinopathy and $8 \%$ have sight threatening DR. ${ }^{10,11}$

Considering the increased prevalence and silent nature of this vision threatening complication of diabetes, the traditional approach where optimum glycemic, lipids and blood pressure control is focused and managed by one specialist and the coexisting complications are either overlooked or managed by different specialists at different places without any coordination, is not desirable.
For proper management of individuals with DM, there is a need for multidisciplinary approach. To achieve this objective, two prong system including referring end and the receiving end of the referral chain was developed. Improvement of awareness in the community and education of the service providers about management of DM resulted in strengthening of referring end of the chain. Establishment of "One window facility" at Al-Ibrahim Eye Hospital (AIEH) reinforced the receiving end of the chain. The present study highlights the results of this system.

\section{METHODS}

A project "strengthening Pakistan's response to Diabetic Retinopathy" was initiated in district Malir, Karachi from April 2014 till March 2020. This observational, cross sectional study was conducted from the data collected during the project, after ethical approval at AIEH. During the project two-pronged system was established in order to strengthen the peripheral (referral end) as well as receiving end of referral the referral system.

Peripheral referral was improved through awareness in the community, enhancing knowledge of service providers and developing linkages with them. The receiving end of the referral was improved by establishing "one window facility" at tertiary eye care center.

One-day training for Medical Officers, General Practitioners and LHW's: To start with one-day structured training was arranged for Medical Officers, General Practitioners, Lady Health Workers and Lady Health supervisors at nearby health facilities in district Malir, Karachi. The main objective of the training was educating health professionals for DM and diabetes related complications with especial reference to Diabetic retinopathy and early referrals of known diabetics for retinal screening to tertiary eye care hospital.

A total of 40 Medical Officers (MO), 55 General Practitioners (GP), 622 Lady Health Workers (LHW) and 24 Lady Health Supervisors (LHS) attended the sessions. At middle of the project, refresher training session was held.

Awareness Raising Sessions: A total of 48 awareness raising sessions were conducted in different locations in district Malir, Karachi by trained health professionals and project team in their catchment areas. Awareness sessions were 
conducted separately for general community (Group-A) and for people with known DM (Group-B).

Group-A: In the sessions for general community, awareness was given about DM, its signs and symptoms, risk group, prevention of DM and key messages on life style modifications like, healthy eating, avoidance of junk foods and importance of physical activity for prevention of DM.

Group-B: In awareness sessions of this group, information was given regarding chronic complications of DM, especially retinopathy, its risk factors like hypertension, blood lipids and blood sugar level and importance of periodic screening of these factors. Importance of SelfMonitoring Blood glucose (SMBG), signs and symptoms of hypo- and hyper-glycaemia, optimal targets for glycemic control, adequate diet, physical activity, and importance of early screening of diabetic complications was discussed.

DR Screening Camps in the Community: To enhance the referral of the patients with DM having diabetic retinopathy, periodic camps were held in various primary health care facilities in district Malir. A total of 85 screening camps were carried out in the community during the project. Direct ophthalmoscopy by optometrist was carried out in all known diabetics attending the camps after dilating the pupil with $1 \%$ Tropicamide. All the individuals having best corrected visual activity of less than 6/18, invisible fundus and any sign of Diabetic retinopathy were referred to the tertiary centre for confirmation, grading and treatment.

Establishment of One Window comprehensive eye care facility for people with DM: AIEH is located in district Malir, a tertiary eye care facility acted as receiving end of the referral chain. One window facility was established in 2014 to cater for the patients with DM. Facility consisted of eight stations starting from registration, visual assessment, fundus photographs, consultation with ophthalmologist, anthropometry measurement, consultation with diabetologist to finally meeting with diabetes educator. At every station, patient's information was directly entered in HIMS software. All patients visiting on their own or referred by various Primary Health care providers were facilitated at an exclusive registration desk. Data was preserved electronically with a unique registration number and source of referral. Demographic data, visual assessment, digital Fundus images on
Non Mydriatic Fundus Camera, slit lamp biomicroscopy with +90D fundus examination was performed. DR (this abbreviation must be used where the term diabetic retinopathy was used the first time. These are basics of writing) diagnosis with grading was made and managed accordingly. DM counselling was done by Certified Diabetes Educator. Individuals with ocular pathologies were referred to respective specialists for appropriate management under one roof. Random blood sugar (RBS) was checked with glucometer, Blood pressure, height and weight of all patients were recorded. Management of DM was done by General Physician. In 2016, diabetologist was introduced to manage DM, screening and basic treatments of diabetic neuropathy (DN), diabetic nephropathy (DN) and diabetic foot (DF). Biochemical tests like RBS, HBA1c, Lipid Profile, Serum Creatinine and Urine DR were made available in laboratory. Patient's status was communicated to the referring source. Follow-up calls were made one day prior to the appointment date.

Statistical Analysis: Data was retrieved from Hospital Information Management System (HIMS) and exported to SPSS version 23.0. Frequencies and percentages were reported in trend chart (year wise) and in graphs for Service acceptance, Referral Source, follow up and treatment uptake for retinopathy.

\section{RESULTS}

Service acceptance: A total of 50,595 patients attended during the study period of six years. New registration was 34685 (68.5\%) with 15910 $(31.4 \%)$ follow ups. Male to female ratio of newly registered individuals was 1.1: 1, with 18245 $(52.60 \%)$ male and $16440(47.40 \%)$ female. Most of the participants, 18363 (52.96\%) were in the age group of 46-60 years.

During first year of the project (2014-15) newly registered individuals with DM were 4414 $(89.5 \%)$ with $518(10.5 \%)$ follow-ups. In the final year (2019-20) new registration was 7916 (62\%) with 4852(38\%) follow-ups (Fig.1).

Referral pattern: During first year of the project, $3867(87.6 \%)$ individuals out of total of 4414 attended on their own, (whereas 291 (6.5\%) were referred by primary health professionals- LHW's $35(0.79 \%)$, MO's $192(4.3 \%)$ and GP's $65(1.4 \%))$, while another $256(5.7 \%)$ were referred through camp screening. 


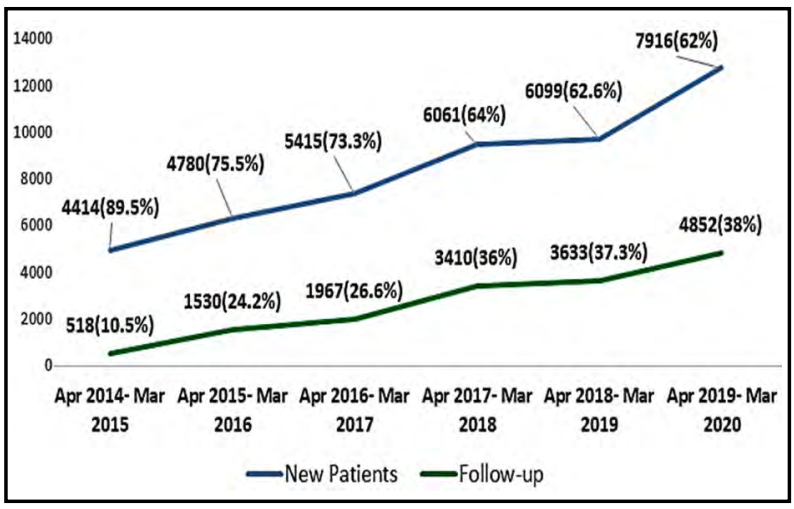

Fig.1: Service acceptance - Frequency of New Registration and Follow ups.

In the last year (2019-20), out of 7916 newly registered patients, 4385 (55.3\%) attended on their own, and $3461(43.7 \%)$ patients were referred by different primary health professionals- (LHW's

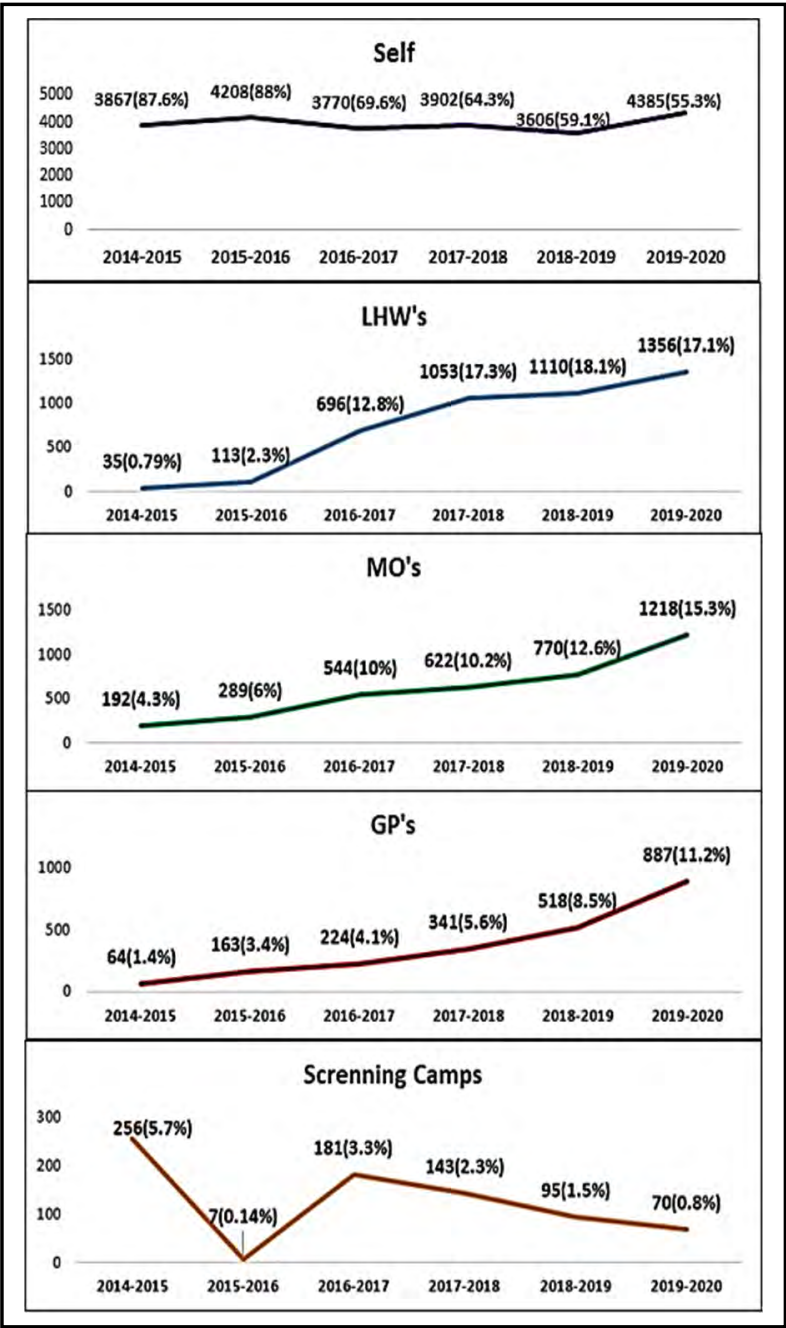

Fig.2: Frequency of patients referred to tertiary care center by different sources.

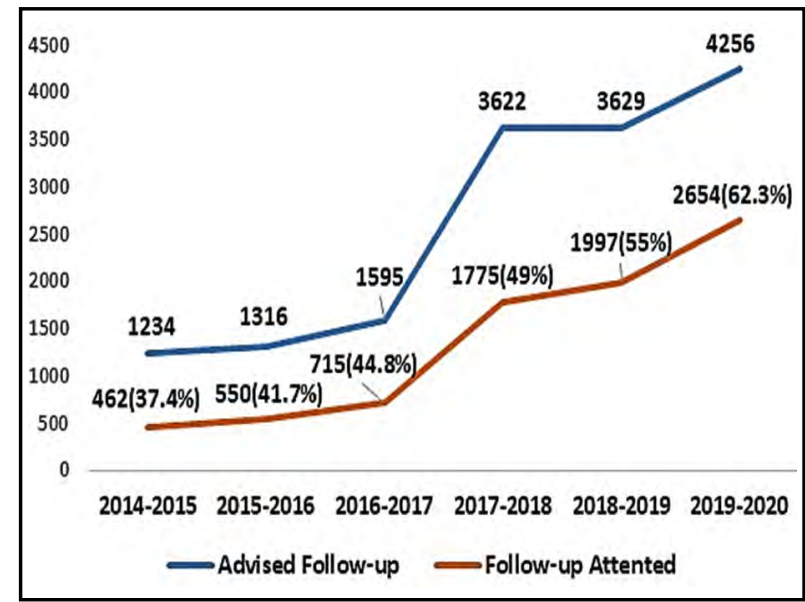

Fig.3: Follow-up Advised and Accepted.

1356 (17.1\%), MO's 1218 (15.3\%) and GP's 887 $(11.2 \%))$, while a further $70(0.8 \%)$ patients were referred through camp screening (Fig.2).

Follow ups: Follow-up is defined as the attendance of an individual within one month of the advised date. During the study period number of followup patients increased from $37.4 \%$ in $2014-15$ to $62.3 \%$ in $2019-2020$, reflecting a rise of $24.9 \%$ over the period of six years (Fig.3).

Treatment Acceptance: At baseline, laser acceptance was $61.24 \%$, intravitreal injection acceptance was $68.56 \%$ and VR surgery acceptance was $13.04 \%$. At 5-year average upto final year, acceptance was found to be $77.98 \%$ in laser, $83.28 \%$ in intravitreal injection and $64.51 \%$ in VR surgery. An increased uptake of all treatment modalities for retinopathy like laser (increased by $16.76 \%$ ), intra-vitreal injections (by $14.72 \%$ ) and vitrectomy (by $51.47 \%$ ) were also observed (Fig.4).

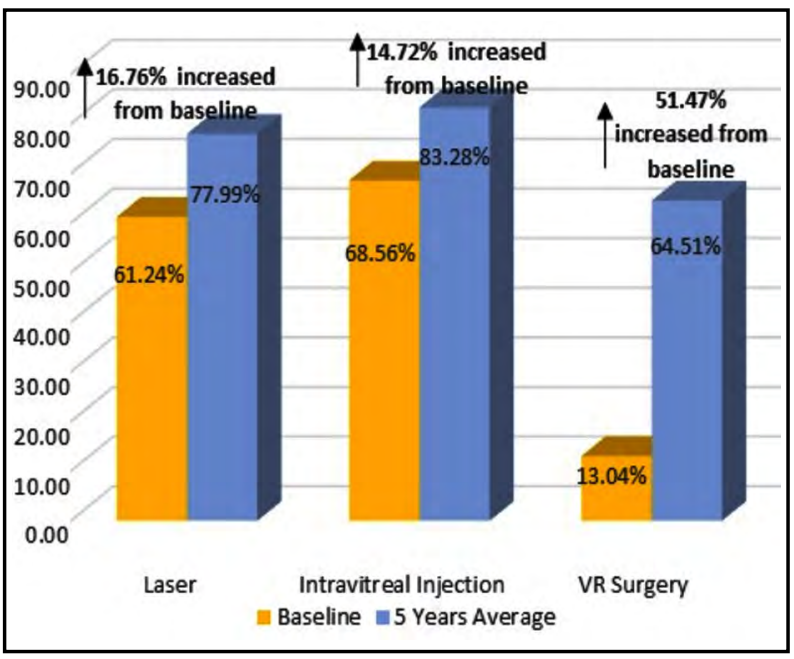

Fig.4: Treatment Uptake for Retinopathy. 


\section{DISCUSSION}

Implementation of two prong system over the period of six years, showed gradual rise in the registration of new and follow-up patients, increased number of successful referrals from primary to tertiary health center and increased uptake of advised interventions.

Referral failure is one of the major obstacles in the delivery of health care services ${ }^{12}$ A sound referral system from primary to secondary / tertiary care health facility is imperative to cater the rapidly increasing number of diabetics and escalating burden on health economy. ${ }^{13}$ The core concept of this design is to encourage the patients to first seek advice at the primary level, and then approach a higher level of care according to the need. This minimizes the costs on the patients and burden on health care provider and higher center resources. However, in most of the cases, patients bypass the primary facilities and self-refer themselves to the higher center. Vice versa, some of the patients who required specialized care failed to get referring advice or did not show compliance to the advice. Thus, a patient in need of expert opinion skips getting the specialized treatment and advice. Even the developed countries had struggled to reduce the referral failure rate that varies widely across the world. ${ }^{14,15}$ British national health services had reported referral failure rate as $12 \%^{14}$ whereas in Western Uganda it was reported to be $31 \% .{ }^{15}$ Various studies originated from Pakistan have identified poor knowledge, limited awareness and indifferent attitude towards the disease as major causes of referral failure particularly for people living in rural and remote areas. ${ }^{16,17}$ These obstacles along with inadequate services, non-coordination amongst the service providers, unaffordability of the patients and absence of counselling at tertiary service level result in lack of treatment acceptance and drop in follow ups. ${ }^{18,19}$

Two prong system was initiated in April 2014 to address these challenges. One prong of the system was dedicated to strengthen the referring end through "Diabetes education program (DEP)". Primary care provider like LHWs, medical officers of the public health sectors and family physicians in the private sector were mobilized through awareness campaign. Knowledge of primary care providers regarding diabetes and its chronic complications was reinforced by the Diabetes
Educator. The primary health care professionals in turn created awareness amongst general community. This strategy improved referrals from primary health care facility to the tertiary level. The results of this approach show gradual increase in registration of new individuals with diabetes at tertiary eye care center. Similar change in frequency of referrals by various referring source (lady health workers, medical officers, general practitioners) was also observed during the study period.

Second prong was directed to strengthen the receiving end of the referral system. One window facility was established to provide comprehensive care to the individual with DM. Diabetes focused registration counter ensured zero loss of the individual with DM attending the tertiary eye care center. It was thought that this will facilitate the referred individuals. Reminder call via help line telephone service a day before the appointment together with counselling resulted in nearly ninefold rise in the number of follow up patients during the period of six years (Fig.1). Counselling by the diabetes educator improved the treatment acceptance.

\section{CONCLUSION}

Implementation of two prong system resulted in improved service uptake, better referral system, enhanced follow-up and increased intervention uptake.

Acknowledgement: This was a collaborative project of Al Ibrahim Eye Hospital Karachi and Sightsavers UK. We are thankful to the Standard Chartered Bank (Seeing is believing project), Sightsavers UK and Diabetes Eye Clinic team of Al Ibrahim Eye Hospital for their support.

Grant Support \& Financial Disclosures: Technical and managerial support to this project is provided by Sightsavers UK while financially this project is supported by Standard Chartered Bank (Seeing is believing project).

\section{REFERENCES}

1. Aravind S, Kumar KMP, Sadikot SM, Nigam A, Bajaj $\mathrm{S}$, Zargar $\mathrm{AH}$, et al. Chronic complications in newly diagnosed patients with Type-2 diabetes mellitus in India. Indian J Endocrinol Metabol. 2014;18(3):355-360. doi: $10.4103 / 2230-8210.131184$

2. Standards of Medical Care for Patients with Diabetes Mellitus. Position statement. American Diabetes Association. Diabetes Care. 2003;26(Supply1):s33-s50. doi: $10.2337 /$ diacare.26.2007.S33 
3. Piyasena MM, Murthy GV, Yip JL, Gilbert C, Zuurmond $\mathrm{M}$, Peto T, et al. Systematic review on barriers and enablers for access to diabetic retinopathy screening services in different income settings. PLoS One. 2019;14(4):e0198979.

4. O'Donnell O. Access to health care in developing countries: breaking down demand side barriers. Cad Saude Publica. 2007;23(12):2820-34. doi: 10.1590/s0102$311 \times 2007001200003$

5. Robin AL, Nirmalan PK, Krishnadas R, Ramakrishnan R, Katz J, Tielsch J, et. al. The utilization of eye care services by persons with glaucoma in rural south India. Trans Am Ophthalmol Soc. 2004;102:47-54.

6. International Diabetic Federation. Diabetes Atlas $9^{\text {th }}$ Edition. Available at: https://www.diabetesatlas.org/en/ sections/worldwide-toll-of-diabetes.html (Accessed date: $10^{\text {th }}$ April, 2021).

7. Yau JWY, Rogers SL, Kawasaki R, Lamoureux EL, Kowalski JW, Bek T, et al. Global Prevalence and Major Risk Factors of Diabetic Retinopathy. Diabetes Care. 2012;35;556-564.

8. Jadon MZ, Dineen B, Bourne RA, Shah SP, Khan MA, Johnson GJ et al. Prevalence of Blindness and Visual Impairment in Pakistan: The Pakistan National Blindness and Visual Impairment Survey. Investigative Ophthalmol Vis Sci. 2006;47(11):4749-4755.

9. Basit A, Fawwad A, Qureshi H, Shera AS. Prevalence of diabetes, pre-diabetes and associated risk factors: second National Diabetes Survey of Pakistan (NDSP), 2016-2017. BMJ Open. 2018;8:e020961. doi: 10.1136/ bmjopen-2017-020961

10. Mahar PS, Awan MZ, Manzar N, Memon MS. Prevalence of type-II diabetes mellitus and diabetic retinopathy: The Gaddap study. J Coll Physicians Surg Pak. 2010;20(8):528-532.

11. Mumtaz SN, Fahim MF, Arslan M, Shaikh SA, Kazi U, Memon MS. Prevalence of diabetic retinopathy in Pakistan: A systematic review. Pak J Med Sci. 2018;34(2):493-500. doi: 10.12669 / pjms.342.13819

12. Afsar HA, Qureshi AF, Younus M, Gulb A, Mahmood A. Factors affecting unsuccessful referral by the Lady Health Workers in Karachi, Pakistan. J Pak Med Assoc. 2003;53(11):521-8. PMID: 14738257.

13. Shera AS, Basit A. PROMPT Team. Pakistan's Recommendations for Optimal Management of Diabetes from Primary to Tertiary care level (PROMPT). Pak J Med Sci. 2017;33(5):1279-1283. doi: 10.12669/ pjms.335.13665
14. Hamilton W, Round A, Sharp D. Effect on hospital attendance rates of giving patients a copy of their referral letter: randomized controlled trial. BMJ. 1999; 318(7195):1392-1395. doi: 10.1136/bmj.318.7195.1392

15. Whitworth J, Pickering H, Mulwanyi F, Ruberantwari A, Dolin P, Johnson G. Determinants of attendance and patient satisfaction at eye clinics in south-western Uganda. Health Policy Plan. 1999;14(1):77-81. doi: 10.1093/heapol/14.1.77

16. Memon MS, Shaikh SA, Shaikh MA, Fahim FM, Mumtaz $\mathrm{SN}$, Ahmad N. An assessment of knowledge, attitude and practices, (KAP) towards diabetes and diabetic retinopathy in a suburban town of Karachi. Pak J Med Sci. 2015;31(1):183-188. doi: 10.12669/pjms.311.6317

17. Memon MS, Fahim MF, Mumtaz SN, Shaikh AS, Shaikh AR. Knowledge, Attitude and practices, (KAP) regarding Diabetes and complications of diabetes among diabetes Type-2 patients visiting a tertiary level eye care hospital in Karachi. Medical Channel. 2015;21(2):11-16.

18. Memon MS, Mumtaz SN, Sheikh SA, Fahim MF. Community Perception and Service Utilization of Diabetic Retinopathy Management Project in Gaddap Town. Pak J Ophthalmol. 2016;32(2):70-77.

19. Memon S, Ahsan S, Alvi R, Fawwad A, Basit A, Shera S, Sheikh SA, Fahim MF. Retinal Screening Acceptance, Laser Treatment Uptake and Follow-up Response in Diabetics Requiring Laser Therapy in an Urban Diabetes Care Centre. J Coll Physicians Surg Pak. 2015;25(10):743-746. doi: 10.2015/JCPSP.743746

\section{Authors Contribution:}

MSM conceived, designed and manuscript writing \& final approval. He is also responsible and accountable for the study.

SA did design of study, manuscript writing \& critical review.

MFF did statistical analysis, Results writing \& critical review of manuscript.

TM did data collection, summarizing of data \& editing of manuscript.

SAS did data collection and methodology writing.

Authors:

1. Dr. Shahid Ahsan, MPhil (Bio), MPhil (NCD), PhD fellow (KU)

Department of Biochemistry,

Jinnah Medical \& Dental College,

Karachi, Pakistan

2. Dr. Muhammad Saleh Memon, FRCS(Eden)

Department of Research,

3. Mr. Muhammad Faisal Fahim, M.Sc. (Statistics)

Department of Physical Therapy,

Bahria University Medical \& Dental College, Karachi, Pakistan

4. Mr. Tauseef Mahmood, M.Sc. (Statistics)

Department of Research,

5. Mr. Sikander Ali Shaikh, M.A (Sociology) Department of Outreach Programs,

2,4,5: Al-Ibrahim Eye Hospital, ISRA Postgraduate Institute of Ophthalmology, Karachi, Pakistan. 